\title{
BRCA Testing in Serbia and Montenegro
}

\author{
Mirjana Brankovic-Magic \\ Institute for Oncology and Radiology of Serbia, Pasterova 14, 11000 Belgrade, Serbia and Montenegro; e-mail: brankovicm@ncrc.ac.yu
}

Key words: BRCA1 and BRCA2 testing, $\mathrm{HBOC}$

Submitted: 30 July 2005

Accepted: 15 October 2005

Serbia and Montenegro is the most recent country to have joined the European integration processes in the Western Balkan region. War has troubled this country for more than ten recent years. Post-war conditions have been as severe as those during the war and include poverty and a lack of new, highly sophisticated technologies and trained professionals as well as appropriate organisation of the health services.

Knowledge of the molecular basis of hereditary disorders has greatly expanded during the last ten years and the principle of genetic testing has been introduced into the clinical practice of Western countries. In these countries, with the USA being among the first, genetic testing for known germline mutations associated with high lifetime cancer risk, for example those involved in familial adenomatous polyposis, introduced the use of consensus data into medical practice. Simultaneously, inventories (maps) of hereditary disorders with frequency and spectra of gene mutations causing particular hereditary diseases were constructed in the majority of European countries. The delay in transition and European integration of our country has reflected also on the status of the investigation of hereditary disorders, including hereditary breast and ovarian cancer (HBOC), resulting in the fact that our country is currently without such a national inventory of its hereditary disorders.

Breast cancer is one of the most common cancers diagnosed in women worldwide and a leading cause of female cancer-related death, despite improvements made in the field of risk factor definition, early detection, diagnostics and treatment of the disease. In central and south-eastern European countries, incidence and mortality trends also show an increment, due to recent changes in risk factors (smoking, age at menarche, age at first pregnancy, oestrogen intake, etc.). In Serbia and Montenegro, breast cancer is the most frequent malignancy and the leading cause of cancer-related death in females as well, with a reported mortality rate of $16.9\left(\mathrm{ASR}_{\mathrm{w}}\right)$ in the year 2000 [1]. Approximately 3,900 new cases of breast cancer are detected in Serbia and Montenegro each year. Twelve hundred of these cases are newly diagnosed at the Institute of Oncology and Radiology of Serbia.

A subset of breast cancer patients with striking family histories is suggestive of Mendelian inheritance of breast cancer risk factors and represents hereditary breast cancer (HBC). About 5 to $10 \%$ of all breast cancers are considered to be hereditary. The most common variant of $\mathrm{HBC}$ is hereditary breast and ovarian (HBOC) syndrome. BReast CAncer genes 1 and 2 (BRCA1/2 genes) with incomplete penetrance have been implicated in about $50 \%$ of all hereditary breast cancers. Discovery of these genes made testing for genetic predisposition for this disorder possible.

Historically, investigations of BRCA genes in Serbia and Montenegro started at the end of the nineties in a collaboration of the Institute for Oncology and Radiology of Serbia with the National Institute of Oncology of Hungary in Budapest [2]. Between the years 2000 to 2003 and 2004 to 2006 joint research programmes with the Greek National Centre for Scientific Research "Demokritos" were developed [3]. Both of these collaborations, especially the one with Greek colleagues, which included the possibility of more than one working visit, strengthened orientation toward BRCA testing at the Institute for Oncology and Radiology of Serbia. In 2004, this institute, with the financial support of the Ministry of Science of Serbia, 
purchased a refurbished $A B I$ PRISM 310 genetic analyser, which enabled complete BRCA testing.

Firstly, it was necessary to set the conditions for blood and tissue samples, as well as DNA storage. As we have already been equipped with liquid nitrogen containers, tissue and cell samples, as well as DNA samples for long maintenance, are stored in liquid nitrogen at minus $196^{\circ} \mathrm{C}$. Secondly, in addition to storage conditions, the most important step towards BRCA testing is the establishment of a Registry of Hereditary Breast/Ovarian Cancer. In the Laboratory of Molecular Genetics, this Registry is presently under construction, mainly based on medical records from the Institute for Oncology and Radiology of Serbia. A genomic DNA bank, consisting of about 190 blood samples from individuals at risk for hereditary breast and/or ovarian cancer, has been constructed. Blood and data of the individuals are included based on established selection criteria [4]. A formal informed consent procedure, approved by the Ethics Committee of the Institute, was initiated in 2004 as part of this process of inclusion. Approximately $90 \%$ of included individuals are breast/ovarian cancer patients.

The majority of families included in our bank are represented by one family member only. Generally, relatives of breast/ovarian cancer patients refuse to be tested - in part because of insufficient awareness of genetic testing and in part because of lack of appropriate procedures for risk reduction (only invasive prevention strategies such as prophylactic surgery demonstrate relevant results in risk reduction). We are also faced with the problem that our people are generally suspicious of new health interventions, especially when they concern genetics. Because of that, during the last year, we have tried to educate our primary care physicians through a series of lectures on hereditary cancer syndromes. A similar educational programme is being developed for lay persons.

So far, complete sequencing of the BRCA1 coding region has been performed in 44 samples. Five mutations were detected in the examined group (Figure $1)$. The presence of 4 known mutations, previously detected elsewhere, has been shown: 185delAG, 3447del4, 5382insC (detected twice) and C61G. The frequency of mutations was $11 \%$, indicating that the examined group was predominantly composed of one or two case families.

Our preliminary investigation found BRCA 1 mutations in 5 families: three in site-specific breast cancer families, one in a breast/ovarian cancer family and one in a family with ovarian cancer only. All identified mutations, except one, were frameshift mutations. Family and molecular data are summarised in Table 1.

As in all countries in transition with very limited resources, the introduction of highly sophisticated and very expensive laboratory techniques into clinical practice is always questionable. Due to the lack of hot spots or recurrent mutations in the majority of geographical areas, except in countries with

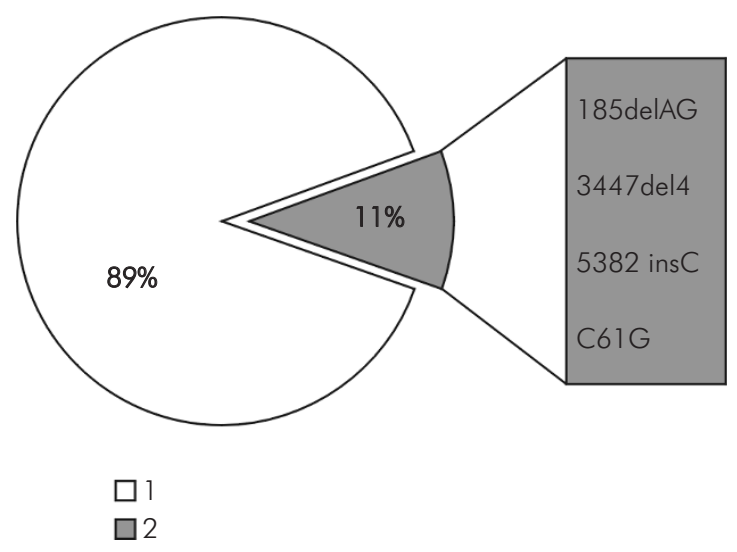

Fig. 1. BRCA1 mutations found in Serbian breast and breast/ovarian cancer families - preliminary results $(n=44) .1$ - without BRCA1 mutations. 2 - with BRCA1 mutations

Table 1. BRCA1 mutations in Serbian breast and/or ovarian families

\begin{tabular}{|c|c|c|c|c|c|}
\hline \multirow[t]{2}{*}{$\begin{array}{l}\text { Family } \\
\text { number }\end{array}$} & \multirow[t]{2}{*}{$\begin{array}{l}\text { Carcinoma of proband } \\
\text { (age of onset, year) }\end{array}$} & \multicolumn{2}{|c|}{$\begin{array}{l}\text { Number of carcinomas } \\
\text { in family }\end{array}$} & \multirow[b]{2}{*}{ Mutation } & \multirow[b]{2}{*}{ Exon } \\
\hline & & Breast & Ovarian & & \\
\hline 4 & Breast $(31,46)$ & 4 & 0 & 5382insC & 20 \\
\hline 6 & Ovarian (22) & 1 & 1 & 3447 del4 & 11 \\
\hline 7 & Breast (42) & 3 & 0 & 5382insC & 20 \\
\hline 14 & Ovarian (69) & 0 & 2 & 185delAG & 2 \\
\hline 29 & Breast (44) & 1 & 0 & C61G & 5 \\
\hline
\end{tabular}


reproductive isolation such as for example Israel and Iceland, direct sequencing of the entire coding region of both BRCA genes is strongly recommended for BRCA analysis. The exception to this rule appears to be Poland where only 3 recurrent mutations represent $90 \%$ of all BRCA1 mutation carriers [5]. In countries, such as ours, without identified founder mutations, whole gene analysis is recommended, which would make population-based screening expensive. We therefore have to answer the difficult question of whether in our country with limited resources it would be wiser to try to identify our recurrent mutations, even if they are not as highly recurrent as for example in Israel and Poland, and to offer testing for these recurring mutations to a large population, or to offer sequencing of the entire coding region of the gene, which is more precise and covers all mutations, to a highly selected population. For our country, this question cannot yet be easily answered due to the lack of population-based studies of hereditary breast and ovarian cancer patients and missing follow-up data of BRCA mutation carriers.

Identification of BRCA mutation carriers, establishment of spectra and frequency of BRCA mutations, especially in breast cancer patients, together with long-term follow-up of mutation carriers, should allow research on the factors affecting penetrance of BRCA gene mutations. Further, systemic BRCA analysis will enable introduction of clinical management for mutation carriers into the clinical practice of Serbia and Montenegro, resulting in real benefit for this highly vulnerable part of the population.

\section{References}

1. Ferlay J, Bray F, Pisani P and Parkin DM. GLOBOCAN 2000: Cancer incidence, mortality and prevalence worldwide. IARC Cancer Base, No 5, Lyon, IARC Press 2001.

2. Papp J, Raicevic L, Milašin J, Dimitrijević $B$, Radulović $S$ and Olah E. Germline mutation analysis of BRCA1 and BRCA2 genes in Yugoslav breast/ovarian cancer families. Oncol Rep 1999; 6: 1435-1438.

3. Konstantopoulou I, Janković R, Raièević L, Ladopoulou $A$, Armaou S, Nikopoulos G, Pandis N, Nasioulas G, Radulović S and Yannoukakos D. BRCA1 and BRCA2 genes mutation analysis in patients with a family history of breast and ovarian cancer. Jugoslav Med Biochem 2004; 23: 271-277.

4. Hampel H, Sweet K, Westman JA, Offit K and Eng C. Referral for cancer genetics consultation: a review and compilation of risk assessment criteria. J Med Genet 2004, 41: 81-91.

5. Gorski B, Byrski T, Huzarski T, Jakubowska A, Menkiszak J, Gronwald J, Pluzanska A, Bebenek M, Fischer-Maliszewska L, Grzybowska E, Narod SA and Lubinski J. Founder mutations in the BRCA1 gene in Polish families with breast-ovarian cancer. Am J Hum Genet 2000; 66: 1963-1968. 